for

\title{
Evidence for Spin Glass Transition in Hexagonal-DyMnO without Substitutional Disorder
}

P. Aravinth Kumar ${ }^{\dagger}$ Arun Kumar, $₫$ Keshav Kumar, ${ }^{\ddagger}$ G. Anandha Babu, ${ }^{\dagger}$ P. Vijayakumar,, S. Ganesamoorthy, ${ }^{\S}$ P. Ramasamy ${ }^{\dagger}$ and Dhananjai Pandey*:

${ }^{\dagger}$ Department of Physics, SSN College of Engineering, Kalavakkam 603110, India

$\$$ School of Materials Science and Technology, Indian Institute of Technology (Banaras Hindu University),

Varanasi 221005, India

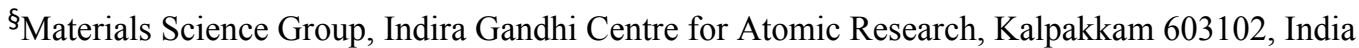

To whom correspondence should be addressed.Email: dp.mst1979@gmail.com 


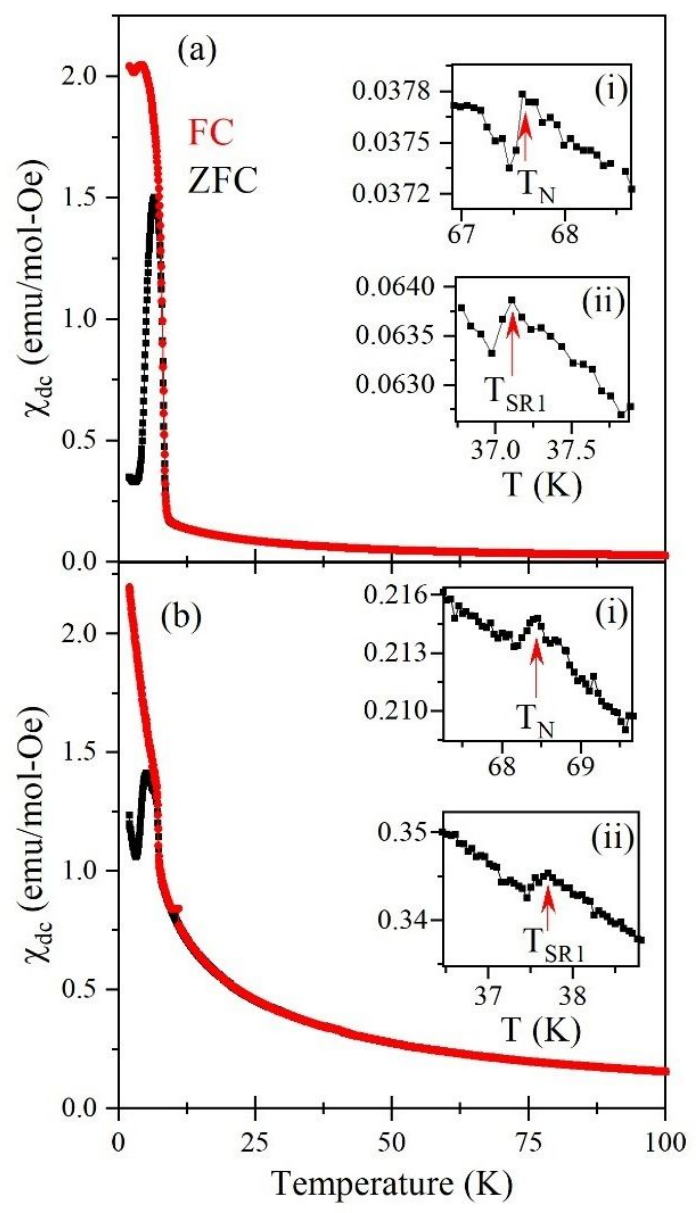

Figure S1. Temperature dependence of dc susceptibility $\chi_{\mathrm{dc}}(\mathrm{T})$ of h-DyMnO $\mathrm{O}_{3}$ crystals measured $\mathrm{H} \| \mathrm{c}$ at $25 \mathrm{Oe}$ field for (a) unannealead and (b) oxygen annealed for 3 days at $1173 \mathrm{~K}$. Insets (i) and (ii) in panels (a) and (b) depict the anomalies corresponding to $\mathrm{T}_{\mathrm{N}}$ and $\mathrm{T}_{\mathrm{SR} 1}$ on a magnified scale. 